\title{
Adaptive Daily Rhythm Atmospheres for Stroke Patients: A Staff Evaluation
}

\author{
Daemen E.M.L., Flinsenberg I.C.M., van Loenen E.J, Cuppen R.P.G., and Rajae-Joordens R.J.E. \\ Philips Research \\ Philips Electronics Netherlands \\ Eindhoven, Netherlands \\ \{Elke.Daemen, Ingrid.Flinsenberg, Evert.van.Loenen,Roel.Cuppen,Roos.Rajae\}@philips.com
}

\begin{abstract}
This paper addresses the evaluation with hospital staff of an in-patient environment that supports patients, family, nursing staff and medical specialists during the recovery process of neurology patients and especially patients recovering from a stroke. We describe the methods that were used to evaluate the Adaptive Daily Rhythm Atmospheres (ADRA) concept. The goal of this evaluation was to gauge expectations against the different phases of the ADRA concept both qualitatively and quantitatively to determine if the different phases are useful, usable and desirable for 1) the healing process of the patient and 2) the workflow of the staff. The results show that the hospital staff expects a positive effect of the different phases of the ADRA concept on the healing process of the patient. In fact the results from the staff of different institutes and with different roles were in agreement on most evaluation criteria. Regarding the clinical workflow, the staff also expects a positive effect for almost all phases. The evaluation results are used to further improve the design of healing environments in general and the ADRA concept in particular.
\end{abstract}

Keywords-component; Adaptive systems, User experience evaluation, Stroke, Healing Environments

\section{INTRODUCTION}

This paper addresses the evaluation with hospital staff of an in-patient environment in which pervasive computing technologies are utilized to design a healing environment that supports patients, family, nursing staff and medical specialists during the recovery process of neurology patients and especial patients recovering from a stroke. We describe the methods that were used to evaluate the Adaptive Daily Rhythm Atmospheres (ADRA) concept with hospital staff and present the quantitative and qualitative results. Two objectives were considered for these evaluations: 1) to improve the patient's healing process and 2) to support the hospital staff in stroke care units. The evaluation results are used to further improve the design of healing environments in general and the ADRA concept in particular.

The healing process is affected by various environmental stimuli in the hospital [1]. For example, there is clear evidence for a positive effect of nature on healing. From literature [2][1][5], we know that access to (rendered) nature views, including the presence of indoor plants, helps to increase the tolerance for pain and reduce the use of pain medication. Also, contact with nature has, to a certain extent, a positive effect on short-term recovery from stress and mental fatigue [2][1], [5], and moreover, it can accelerate the physical recovery from illness
[5], [6], for example from elective cervical and lumbar spinal surgery. Not only nature views, but also exposure to daylight is found to be an important factor in the recovery process. Patients exposed to sufficient daylight are less stressed and seem to need less pain medication [7]. Bright (artificial) daylight exposure during day-time and avoidance of too much light exposure during night-time helps to sleep better at night [8] and to feel more energized during the day [9]. Especially a deep restorative and undisturbed sleep is of high importance for a fast recovery process in patients.

Lately, a great number of hospitals have chosen to provide more single bedded rooms to patients because there is growing confidence and evidence that these rooms have a positive effect on patients. Private patient rooms have become the industry standard in the United States because they better facilitate patient care and management, afford greater therapeutic benefits for patients [10], [11], [12], and may reduce the rate of hospital-acquired infections, although conflicting results have been found. Also in Europe, there is a tendency to provide only single rooms to patients for these reasons.

Today, most hospital patient rooms remain highly institutionalized environments that confine patients to an artificial and largely alien world and provide little opportunity to adapt the environment to individual patient requirements. To gain more insight into the actual needs of patients in hospital rooms, we conducted exploratory site visits at several hospitals.

\section{IDENTIFICATION OF INITIAL USER REQUIREMENTS}

Although we performed extensive desk research, we did not have enough knowledge about stroke patients and their medical environment, the neurology ward. What do these patients and clinical stakeholders experience? What are their feelings and emotions? What do we know about the neurology ward? To answer these questions, we carried out contextual research in two neurology departments and rehabilitation centers. This contextual research was set-up as exploratory site visits. These visits provided us a way to gather first-hand knowledge on how care is currently delivered and experienced by all stakeholders involved, i.e., everybody with a task in and around the patient's bed, and on the context in which these activities take place. 


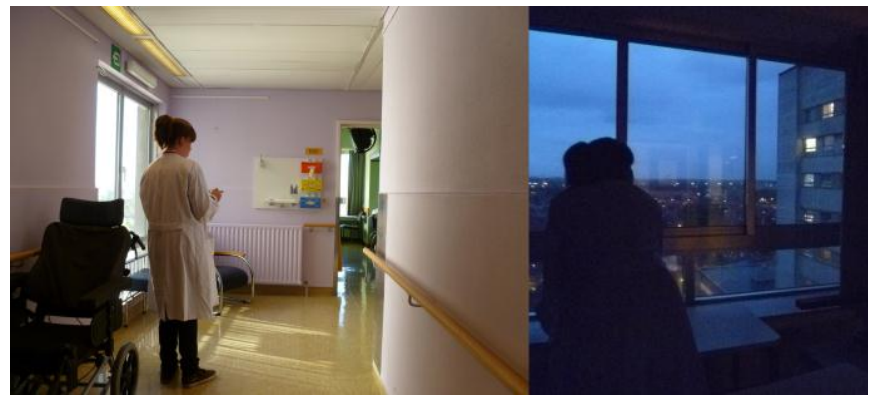

Figure 1: Doing observations day and night.

Our major focus for these visits was on the environment of single patient hospital rooms and on all relevant aspects that could be instrumental to enhance the healing process by means of context-related adaptations in the environment. For this, we started with a guided tour, followed by the main field research methods: shadowing stakeholders, doing observations (see Figure 1), environmental analysis, and interviewing stakeholders. Immediately after each activity, the data were analyzed and visualized in an experience-flow [9]. We concluded both field studies with a multi stakeholder session where we confronted all the stakeholders with the initial user requirements that were derived as outcomes of our analyses. This knowledge and understanding resulted in a collection of initial user needs and requirements that could be used to develop potential solutions addressing the needs of both patients and staff. This process and the methods that were used to collect these initial user needs and requirements are reported in [13].

A major finding was that stroke patients are at high risk to suffer from disorientation, confusion and delirium. This implies that they have a strong need for orientation in time and place. Furthermore, they need a clear and strict daily structure and support to maintain a healthy sleeping pattern. These observations are confirmed by the medical staff of neurology departments and rehabilitation centers for stroke, brain injury, and brain infection. However, the current conditions in hospitals are static regardless of the patient's needs or care activity schedule. Another finding was the importance of establishing the right balance between a clinical environment and a personal environment for all people involved. For example, patients and their family need personal spaces for privacy purposes and to escape, re-energize, and relax from all invasive and emotionally draining activities, but the room should also provide a clinical environment whenever a medical action is to be performed. Furthermore, patient rooms need to facilitate an optimal working environment for the hospital staff. This issue is particularly relevant given that stroke patients typically stay longer in the hospital than many other patient groups.

These findings from the site visits combined with the established healing effects from literature led to a total of 11 healing issues and opportunity areas. These provided a base to generate a large number of application concepts to improve the healing process. In total 21 concepts were then developed addressing these areas. After an internal evaluation of these concepts a subset of about ten concepts was proposed to the staff of four hospitals. Based on their input four concepts were selected for further development. These adaptive healing room concepts have now been prototyped and are installed in our laboratory environment for further evaluation and iterative design and development.

\section{ADAPTIVE DAILY RHYTHM ATMOSPHERE}

One of these concepts that is currently installed is the Adaptive Daily Rhythm Atmosphere (ADRA). ADRA supports the daily rhythm of the patient by generating dedicated multisensory atmospheres for different phases throughout the day that consist of manipulating light, audio, and video parameters. Where needed, the atmosphere can adapt to specific interrupts and visits, for example, when a doctor is visiting or a cleaner starts working. It is assumed that by using ADRA, the potential negative effects of the rigid environmental conditions in the healing room will be alleviated, because the system provides a daily rhythm atmosphere that is in sync with and optimized for the patient's needs and the care agenda, and that intelligently adapts to deviations thereof. To implement this ADRA concept, we created a context-aware system based on sensor input concerning person location, patient activities, and time of day. For technical details on the context-aware system and its implementation, we refer to [13].

The ADRA approach explores how an context-aware system can be used to actively adapt the local environmental conditions to changing patient needs throughout the day during their stay in the hospital, and is therefore different from previously proposed systems which focus on providing the hospital staff with proper information; see for example [15][14]-[20]. In this way, ADRA also goes beyond previous patient-focused work in the hospital, which mostly aims at patient monitoring, and handling of electronic medical records or personal health records, see for example [21]-[26].

Although the application focus of our system is different from previously proposed systems, some of the technologies developed for other systems can be utilized. For example, existing person tracking technology, patient monitoring techniques, and context aware system design principles can be applied. The challenge is to create a robust yet flexible system that achieves our stated objectives while taking advantage of state-of-the-art technology.

\section{A. Details ADRA concept}

The goal of the Adaptive Daily Rhythm Atmosphere (ADRA) is supporting the daily rhythm of the patient. Therefore ADRA will generate dedicated multisensory atmospheres (e.g., light, audio, and video) for different phases throughout the day.

In order to realize the multisensory atmospheres, we have installed an artificial skylight, a light cove behind the patient bed, electrically operated window curtains, and a patient wall in front of the patient containing two RGB light coves, and three multi-media screens. The middle screen is used to show nature views (still images, slide shows and movies), the left screen is used to show time and location information, and the right screen is used to show personal photos and drawings. Figure 2 gives an illustration of the set-up. 


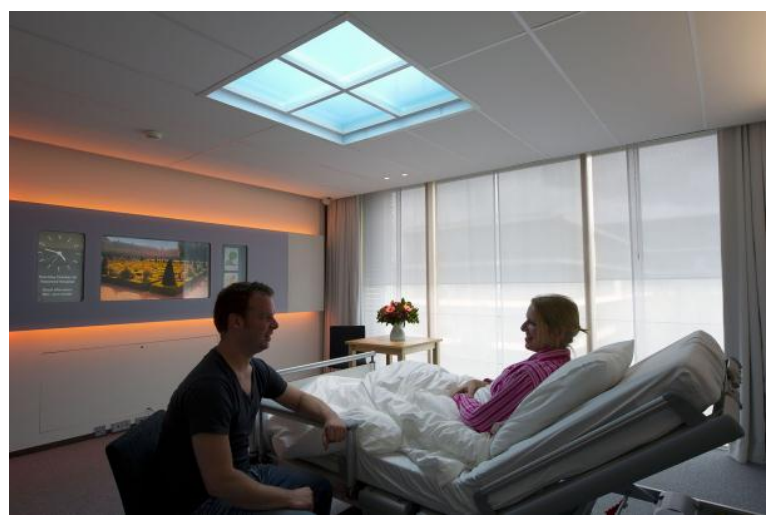

Figure 2: Illustration of the laboratory set-up (Visitors phase).

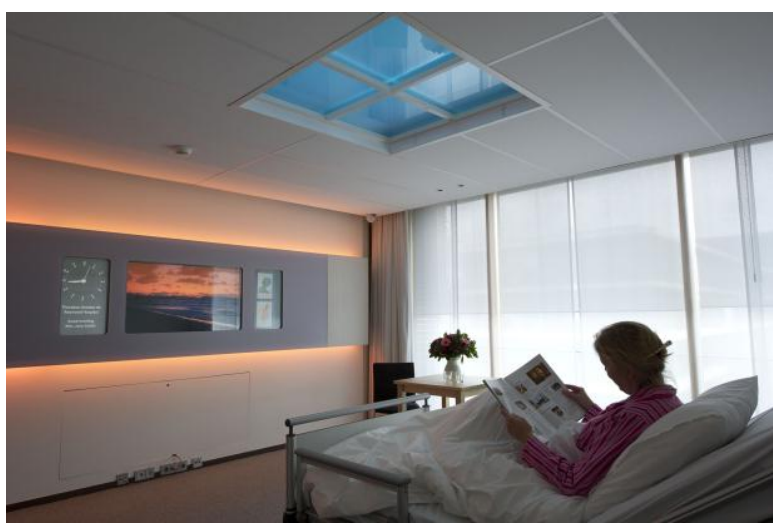

Figure 3: Going to bed.

The following 8 phases are identified in the system: 1) Waking up, 2) Breakfast, 3) Clinical care, 4) Lunch, 5) Rest, 6) Visitors, 7) Going to Bed, and 8) Sleep. Each phase has a defined time frame during which it is active. The used times are determined based on the actual timing used by one of the hospitals we visited. Hospitals can of course adjust the scheduling to their or their patients' needs. For a video showing the different phases, please consult [27]. We will now shortly describe the scenario for the different phases.

\section{1) Waking up (07:30-08:00)}

At the start of the waking up phase the room is still dark and all lights, video, and audio are turned off. The room slowly prepares the patient for a gentle wake up by slowly increasing the light intensity in the room, such that it mimics a sunrise. When the light intensity is at its final level, a nature view slide show appears on the screen in front of the patient and an audio file with singing birds is played (Figure 4). Just a few minutes before eight o'clock, the window curtain furthest from the patient's head will automatically open to allow natural outside daylight entering the room to light up the room without shining directly in the face of the patient. One minute later, the other window curtain follows. By the end of the waking up phase, the patient should be awake and be ready for breakfast and personal care.

\section{2) Breakfast (08:00-10:00)}

Because stroke patients sometimes have trouble with swallowing, a still picture of a nature view replaces the wakeup content to avoid distraction. For the same reason the audio is muted. To strengthen the natural circadian rhythm, and to gently prepare the patient for the clinical care phase to come, the artificial light intensity is slowly increased during breakfast. The light is also designed to be suited for personal care activities like washing and getting dressed.

\section{3) Clinical Care (10:00-12:00)}

During clinical care the light intensity reaches its maximum level. This light in the room allows the doctors to examine the patient and the therapists to give therapy (Figure 5). The light is also designed to be activating, and to have characteristics expected to increase alertness, aimed at helping patients to better remember the doctor's instructions and complete their therapy. Furthermore, sufficient light exposure during the day can reinforce a healthy sleep rhythm. During this phase nothing will be shown on the screens and privacy glass on the patient wall is turned opaque to hide the screens.

\section{4) Lunch (12:00-13:00)}

Just like during breakfast, a still picture of a nature view is shown and the audio is muted, to allow the stroke patient to fully concentrate on eating. The light intensity is slowly reduced from its maximum level to a level similar to that during breakfast.

\section{5) $\operatorname{Rest}(13: 00-15: 00)$}

During rest one curtain closes to darken the room. The other curtain remains open to make a clear distinction between the afternoon rest and sleeping at night. During rest, the patient wall displays a nature view with related nature sounds. The sound helps to mask noises in the corridor, and the relaxing nature video should make the patient feel relaxed and reduce pain perception [3].

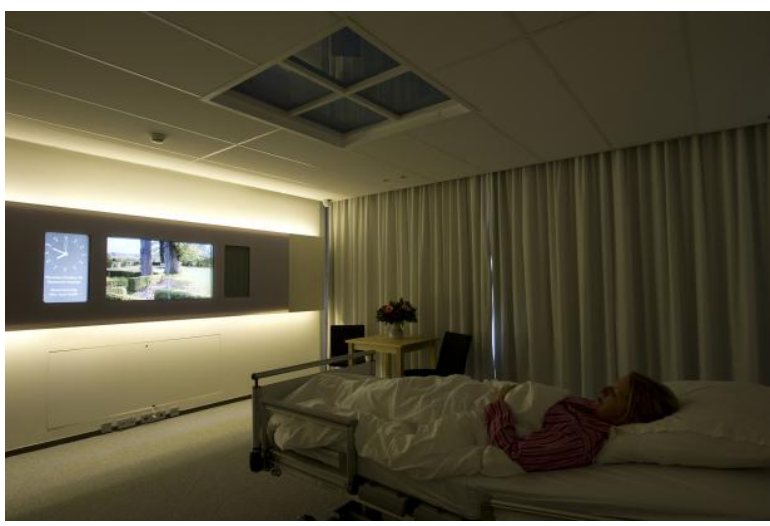

Figure 4: Waking up phase.

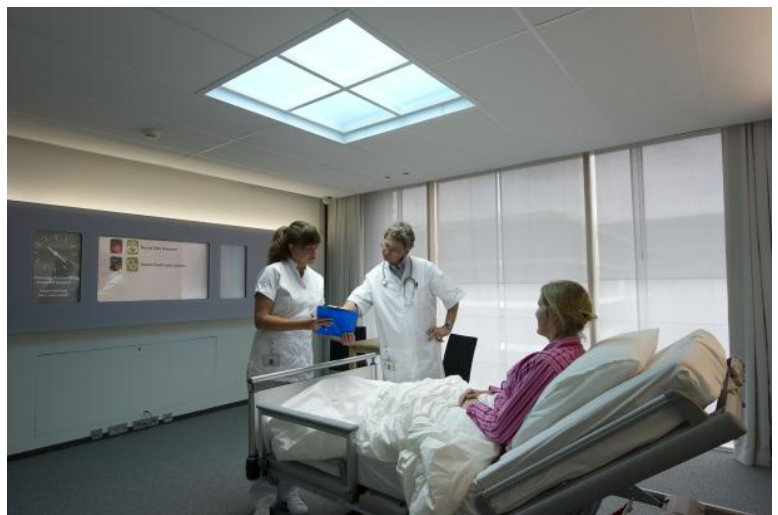

Figure 5: Doctor's visit during clinical care phase. 


\section{6) Visitors (15:00-20:00)}

In contrast with the clinical care setting, the visitor setting is cozy. We achieve this by creating colored light from the light coves and generating a nature picture on the screen. We know from other studies, that visitors tend to stay longer in patient rooms with a pleasant and cozy atmosphere. In general, patients benefit from social contacts (Figure 2).

\section{7) Going to Bed (20:00 - 22:00)}

The room slowly prepares the patient for sleep by slowly reducing the light intensity and removing most blue light from the room, as blue light especially is known to suppress melatonin production and impact circadian rhythm. A movie of a sunset is shown and again restful sounds are played to mask the sounds of the hallway (Figure 3).

\section{8) Sleep (22:00-07:30)}

All the lights and the screens are turned off, except the orientation screen which stays available during the night at very low intensity. Sufficient darkness during the night reinforces a healthy sleep pattern. When a nurse enters the room she is automatically detected and a dimmed light condition is created that just allows the nurse to visually inspect the patient and check medical parameters if necessary. The lights automatically turn off after the nurse has left the room. For a doctor, a high light intensity condition is used because the presence of a doctor in a patient room at night indicates the patient has a severe medical problem.

\section{HosPitAl STAFF EVALUATION}

The goal for the evaluation of the different phases of the ADRA concept with the hospital staff was to obtain qualitative and quantitative feedback to determine if the different phases are useful, usable and desirable for the healing process of the patient and workflow of the hospital staff. These evaluations were set-up as pilot tests in a laboratory setting to refine the concept and to prepare for a clinical trial. Since clinical trials are very time consuming and labor intensive, it is important and extremely valuable to pre-test all the concepts in the laboratory to optimize the procedures for the clinical trial and to minimize technical and usability risks. In addition, by conducting this laboratory study we also could obtain feedback from different hospitals and departments that are involved in the overall project, whereas with a clinical trial we would only get results from staff and patients from one particular hospital department and setting. Hence the laboratory study allows us to judge the differences between departments and hospitals.

\section{A. Set-up of the laboratory hospital room}

Figure 6 shows the set-up of the laboratory hospital room that was used for the evaluation. The task of the moderator was to lead the session. Two note takers participated to write down all the qualitative feedback the participants gave. A maximum of 4 participants took part in the evaluation process at a time. Participants were seated next to the patient bed, so they had a similar view as the patient would have. The moderator was standing on the left side and two note takers took place at the table on the right. At this position they were not blocking the view of the participants.

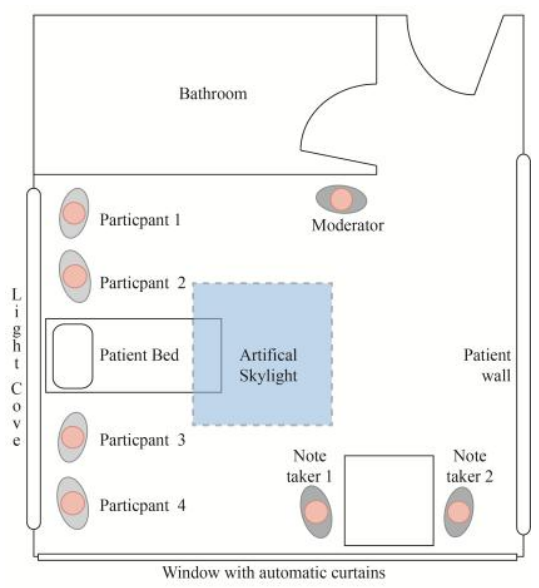

Figure 6: Set-up.

\section{B. Participants}

We invited medical staff from different departments from different hospitals, being neurology, rehabilitation and geriatric departments to our laboratory environment. They came in groups of 2-4 persons per session as a single group for each hospital department. The group session was selected because we hoped to learn from the discussion and interaction between the different staff members that are responsible for different functions in the care process (e.g., nurses, doctors, managers). Furthermore, since the entire evaluation session takes on average about 3 hours, the staff members were more willing to participate as a group then they were individually. The participants came from four neurology departments ( 9 nurses, 5 neurologists and 3 department managers), three rehabilitation departments (4 nurses, 2 rehab specialists, 1 department manager and 2 therapists), and one geriatric department (2 nurses, 1 geriatric specialist, 1department manager). We had 30 participants in total who had on average 13 years of experience.

\section{Methodology}

After signing the informed consent, the group of participants was taken to the laboratory environment where they were introduced to the ADRA system and could experience every phase of the ADRA system. Then, the participants were seated next to the patient bed so they had a similar viewing angle as the patients. The participants received a booklet with questionnaires. The items on this questionnaire addressed the influence on the healing process (i.e., the possible effect the phase has on the healing process of the patient, meaning faster recovery, better sleep and enhanced well-being) and influence on the workflow (i.e., the possible effect of such a phase on the working activities of the staff in the ward). After experiencing every phase they rated the phase anonymously on a 7 point Likert scale. In addition to rating the phase in the therefore designed booklets, they were also asked to motivate their ratings in writing. Subsequently, a focus group discussion took place. During the discussion the two 


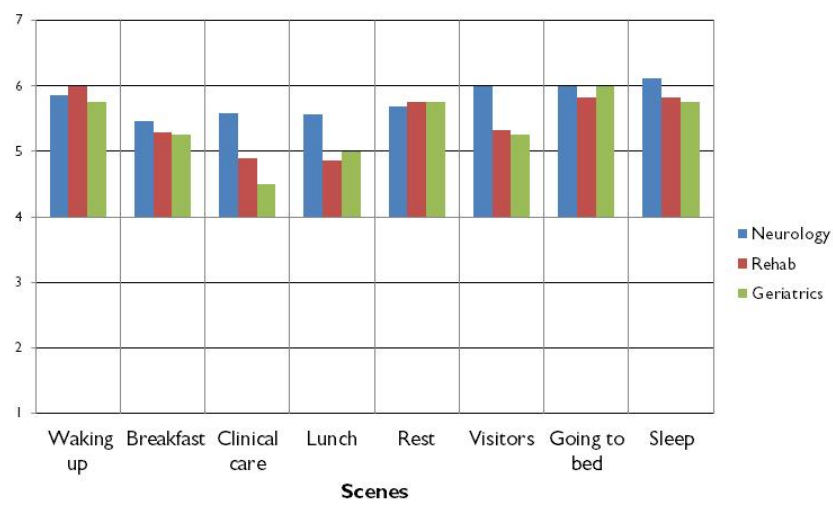

Figure 7: Impact on Healing according to department.

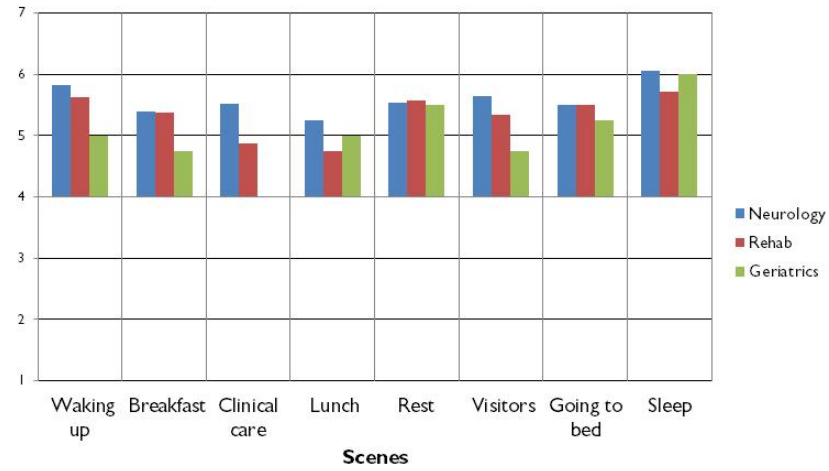

Figure 8: Impact on Workflow according to department.

note takers wrote down all the comments. Afterwards the quantitative results were analyzed with the non-parametric Kruskal-Wallis test.

\section{Quantitative findings}

First we looked at the data per department. The three different departments (neurology, rehabilitation and geriatrics) rated all the implemented ADRA phases as having a positive impact on healing (see Figure 7). They also expect all phases to have a positive influence on workflow (see Figure 8), except for the participants of the Geriatrics department who rated the effect on the workflow of the hospital staff for the clinical care phase neutral. There were no significant differences in expected impact on healing between different departments, except for the phase Visitors. As can also be seen in Figure 8, the neurology department rated this phase significantly higher than the rehab centers and the geriatrics department $(\mathrm{p}=0.028)$. According to the qualitative feedback given, the stakeholders from the neurology departments were convinced that by providing such a Visitors phase relatives would visit the patient more and also stay longer with the patient. The staff finds this very important because patients can be helped by their relatives to get dressed, eat, or even with rehabilitation exercises. Because of the severe conditions the patients are in, this is a great help for the nurses in a neurology ward.

Next we looked at the data per function. Figure 9 and Figure 10 show that all the different functions, i.e., department manager, geriatrician, neurologist, nurse, rehabilitation specialist, and therapist, rated the impact of all phases on Healing and Workflow as positive, except for the therapists who rated the

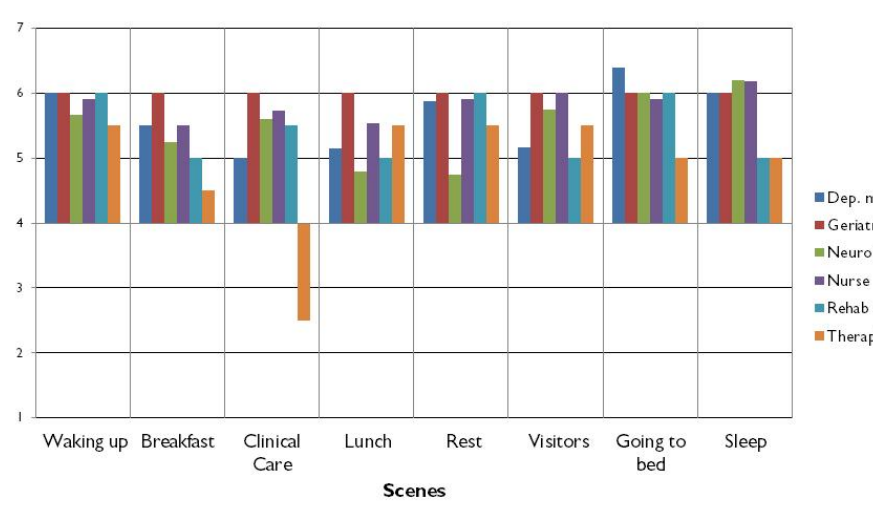

Figure 9: Impact on Healing according to Function.

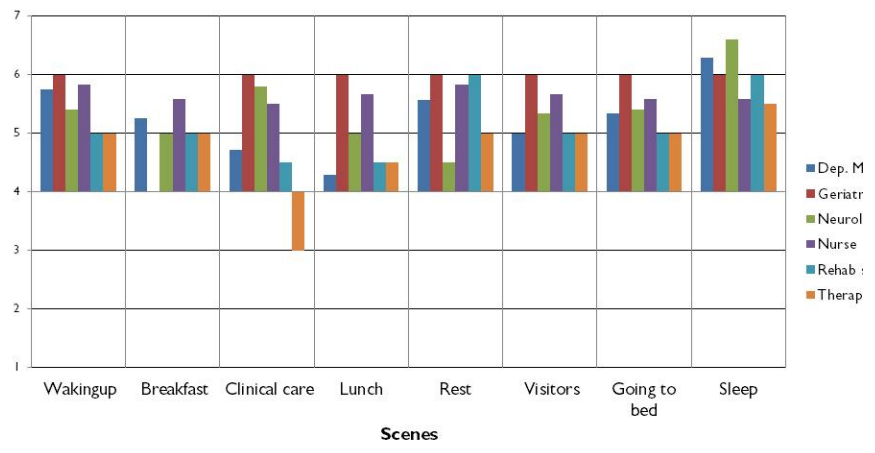

Figure 10: Impact on Workflow according to Function.

impact of the phase clinical care on healing and on workflow negative (mean 2.5 for healing and mean 3.0 for workflow). They preferred a warmer atmosphere to build up a relation with their patients. There was no significant difference between functions for effect of the phases on healing. There was a significant result for the phase Lunch on workflow $(\mathrm{p}=0.006)$.

\section{E. Qualitative findings}

The qualitative feedback of the participants was further elaborated in the focus group sessions. According to the hospital staff members the ADRA phases were a good example of a patient centric approach. For example, one of the participants expressed it as follows: "It is tailored around the patient. In the past we tailored everything to the doctor, but it is very important to tailor it to the patient." To illustrate this, consider the waking up phase. From the context studies we learned that nowadays when nurses enter the room in the morning, patients are often still asleep and nurses need to wake them. Patients are thus woken up very abruptly, and feel their privacy is invaded and therefore can be grumpy and even aggressive. According to participants the ADRA waking up phase will prevent this, and patients will not be woken abruptly anymore when nurses enter their room. Therefore the participants and especially the group of nurses believed that the waking up process with ADRA would cause the patient to be less aggressive, angry and grumpy. Furthermore, it would also be beneficial for the workflow because nurses don't need to wake patients anymore and patients are expected to be more cooperative. As one of the department managers formulated it: "With this phase you are patient friendly without doing 
anything. It is like saying "good morning" without saying it." The participants indicated also that the 30 minutes might be too long to wake-up, but that this needs to be tested with patients in a clinical trial.

The participating hospital staff members believed that the consistent schedule imposed by ADRA would have a positive effect by giving more structure in the day for the patients. They assume that it allows the patient to identify the rhythm of the day and therefore they will anticipate what is coming, for example, breakfast or lunch. As one of the nurses mentioned: "I believe it is nice that the patient gets the time to prepare - now he doesn't get that time..." According to the staff the use of an imposed rhythm would reduce the likelihood that patients reverse their day and night rhythm. As one of the neurologists said: "The patient is stimulated to stay awake in the morning, this is good for his day and night rhythm.". Therefore the detection of the presence of the nurse during the night was also liked. Such a 'night light' will not wake the patient and is enough to assist the nurse. Staff thought the light was quick, effective and workable. One of the department managers said:

"The nurses are assisted by the automatic light - no fumbling with the buttons and accidently turning on the wrong light, therefore the patient is not disturbed and will not be woken." Staff recognized many more of the opportunities of using sensing of the presence of staff and patients as attractive, for example the option to combine tracking of patients with a nurse alert system or automatically turning on the light when detecting that a patient leaves the bed to go to toilet.

Hospital staff also indicated that the room was giving the right number of stimuli over the day. For example, while eating concentration is needed, so staff believed that the breakfast and lunch phase were ideal for patients because there was no audio or video distraction and therefore patients can better concentrate on eating. Also in the clinical care phase we provided a distraction free environment with a light boost of the artificial skylight. Staff believed that the light would work as an activating agent and that this is beneficial for the therapy. As one of the department managers mentioned: "Clinical examination and nurse activities are enhanced by a patient that is more awake.". On the other hand staff indicated that the clinical phase takes too long and therefore becomes boring, and that the atmosphere is too cold. Staff members suggested activating the phase only when the doctor and/or therapist are present in the room, and show a nature image and photos only when staff is not present. The two therapists indicated that they would like to have a cozier atmosphere to enable building a trustworthy relationship with the patient.

Hospital staff also believed that the ADRA concept would result in a behavior change on their part and that the system would help them to keep track of the time of day. As one of the nurses indicated: "As a staff member you are made aware by the atmosphere that this is for example resting time, so you will also adapt your behavior to this". They also indicated that at some moments such as in an acute situation, they should have the possibility to overrule the system and brighten the light.

According to the participants the room would not only be beneficial for the patients but also for the family members. One of the nurses indicated: "A lot of family members think a hospital is scary - this will reassure them and make them stay longer. This is beneficial for the patient". The Visitors phase will also evoke visitors to be calmer by presenting a serene atmosphere. This will be beneficial for the wellbeing of the patient, for example, as one quotes: "It is like a home atmosphere in the room - cozy and enjoyable light.". The participants also suggested that the time of visiting hours could be adapted to the severity of the condition of the patient. If the staff can shorten the visiting hour atmosphere in this way, visitors stay less long when a patient is very ill. They also indicated that it would be nice if the room could know when there are no visitors present and could show a nature video on the screen.

The hospital staff, however, was missing options for personalization and the possibility for patients to control certain settings of the room. According to them, patients had to be able to choose the images, sound and light settings, albeit to a limited extent, in order to give them control over the patient room. The amount of control should be tuned to the patient capabilities, because depending on the severity of the stroke and their recovery progress, patients may or may not be able to handle certain control options. They also believed it was important to give patients the possibility to watch TV on the screen in front of the patient. Furthermore, the staff indicated that the system should be designed around the hospital program and not the other way around. For example 'Waking up' should be staff driven and not patient driven as one of the neurologists said: "My hospital is not a hotel.". So a good balance between personalization and generalization is necessary.

\section{CONCLUSION AND DISCUSSION}

These results show that hospital staff expects a positive effect on the healing process of the patient of the different ADRA phases that represent the different phases of the hospital day. In fact the staff members from different healthcare institutions and with different professional roles agreed on most aspects. In addition, the staff also expected a positive effect for almost all phases on the efficiency of the clinical workflow. This is a very promising result. It shows that careful consideration of both patient and staff needs emerging from context studies enables the design of patient centered solutions that do not negatively impact staff workflow, and can even improve it.

Our context-aware system has been designed to be able to handle dynamic changes to the phase timing, see [12]. The study explored staff attitude to such intelligence aspects of smart patient rooms. From the staff feedback there was a wish not to adapt the phases dynamically to the individual characteristics of the patient (e.g. early or late riser). However, dynamic adaptation to the care being 
provided (e.g. doctor visits) was desired. We also recorded staff interest in other applications of context awareness in patient rooms, such as intelligent lighting to improve patient safety. It shows that the range of relevant applications for smart environment technologies in hospitals extends beyond more extensively studied themes such as location based information distribution. It is interesting to investigate if patients agree with these findings, what their attitude is towards an imposed day schedule, and which further needs for dynamic adaptation emerge from a clinical trial.

Although there is a trend towards single patient rooms, the described system can also be applied to multi-patient rooms, especially when the timing of the different phases is controlled by the hospital staff and not by the patients themselves. In case the phase timing does need to be adapted to, or by, each individual patient, additional research on the embodiment is needed regarding creating personal spaces in multi-patient rooms and handling conflicting patient requirements. Another interesting research direction is to explore the possibilities of using the imposed day schedule in the next phases a patient may go through in his healing process: the rehab center and the home of the patient.

The hospital staff evaluation in the laboratory setting gives an indication of the likely impact of the ADRA phases on the healing progress of patients. Furthermore, this laboratory evaluation of the concepts was an important step that enabled to improve the shortcomings of the current concept before starting clinical trials. In addition, we generated feedback from different departments from different institutions which suggest that they all see similar added values of the ADRA concept. In contrast, with a single-site clinical trial we would only obtain feedback from one hospital department and we would not be able to generalize the findings to other institutions. In addition the laboratory evaluations helped to make hospitals enthusiastic to participate in a clinical trial because they could experience the concepts first hand and see the added value.

As mentioned in the introduction, the goal of the evaluation was to evaluate the ADRA concept with hospital staff. With regard to future work, the other important stakeholders of the ADRA concept, the patients and family, need to be involved. As a next step to prepare for a comprehensive clinical trial, the concept will be evaluated with ex-patients and family in the laboratory setting. The goal of this test then is to assess their opinions, feelings and attitudes towards the ADRA phases in order to gain understanding of their expectations and determine whether the ADRA system could improve the experience and wellbeing of patients during their hospital and rehabilitation period. Next we will conduct clinical trials in a real hospital setting to test the Adaptive Patient Room with patients.

\section{ACKNOWLEDGMENT}

We thank all patients, medical staff, researchers and designers that have contributed to defining and validating the issues, concepts, and solutions proposed in this work. Furthermore, we thank all who have contributed to improving this paper.

\section{REFERENCES}

[1] R.S. Ulrich, The role of the physical environment in the hospital of the $21^{\text {st }}$ century: a once-in-a-lifetime opportunity, Robert Wood Johnson Foundation, 2004.

[2] G. B. Diette, N. Lechtzin, E. Haponik, A. Devrotes, and H.R. Rubin, "Distraction therapy with nature sights and sounds reduces pain during flexible bronchoscopy", Chest, vol. 123(3), pp. 941-948, 2003.

[3] B. Grinde, and G.G. Patil, "Biophilia: does visual contact with nature impact on health and well-being?", International Journal of Environmental Research and Public Health, vol. 6(9), pp. 2332-2343, 2009.

[4] R.S. Ulrich, "View through a window may influence recovery from surgery”, Science, vol. 224(4647), pp. 420-421, 1984.

[5] M.D. Velarde, G. Fry, and M. Tveit, "Health effects of viewing landscapes - landscape types in environmental psychology", Urban Forestry \& Urban Greening, vol. 6 (4), pp. 199-212, 2007.

[6] J.M. Walch, B.S. Rabin, R. Day, J.N. Williams, K. Choi, and J.D. Kang, "The effect of sunlight on postoperative analgesic medication usage: a prospective study of spinal surgery patients", Psychosomatic Medicine, vol. 67(1), pp. 156-163, 2005.

[7] T. Wakamura, and H. Tokura, "Influence of bright light during daytime on sleep parameters in hospitalized elderly patients", Journal of Physiological Anthropology and Applied Human Science, vol. 20(6), pp. 345-351, 2001.

[8] T. Bringslimark, T. Hartig, and G.G. Patil, "The psychological benefits of indoor plants: a critical review of the experimental literature", Journal of Environmental Psychology, vol. 29(4), pp. 422-433, 2009.

[9] A. BaHammam, "Sleep in acute care units", Sleep and Breathing, vol. 10(1), pp. 6-15, 2006.

[10] Chaudbury, "Nurses' perception of single occupancy versus multioccupancy rooms in acute care environments: An exploratory comparative assessment", Applied nursing Research , vol. 19, pp. 118125, 2006.

[11] I. Glind, S. Roode, and A. Goossensen, "Do patients in hospitals benefit from single rooms? A literature review", Health Policy, vol. 84, pp. 153$161,2007$.

[12] K. Dijkstra, "Understanding healing environments: Effects of physical environmental stimuli on patients' health and well-being", University of Twente, 2009.

[13] E.M.L Daemen, R.P.G. Cuppen, I.C.M. Flinsenberg, E.J. van Loenen and R.J.E. Rajae-Joordens, "Contextual research for healing patient rooms design patient experience flow studies in neurology departments", Proceedings of the First European Conference on Design 4 Health 2011, Sheffield UK, 13 - 15 July 2011.

[14] I. Flinsenberg, R. Cuppen, E. van Loenen, E. Daemen, and R. RajaeJoordens, "Context-aware system for neurology hospital wards" , Wireless Mobile Communication and Healthcare, Lecture Notes of the Institute for Computer Sciences, Social Informatics and Telecommunications Engineering, vol. 83, pp. 366-373, 2012.

[15] J.E. Bardram, "A novel approach for creating activity-aware applications in a hospital environment", Proc. INTERACT 2009, Part II, LNCS 5727, pp. 731-744, 2009.

[16] J.E. Bardram, "Applications of context-aware computing in hospital work - examples and design principles", Proc. ACM Symposium on Applied Computing, pp. 1574-1579, 2004.

[17] J.E. Bardram, "Hospitals of the future - ubiquitous computing support for medical work in hospitals", Proc. Hospitals Workshop Ubihealth, 2003.

[18] D. Sanchez, M. Tentori, and J. Favela, "Hidden markov models for activity recognition in ambient intelligence environments", Proc. Eighth Mexican International Conference on Current Trends in Computer Science, IEEE Computer Society Washington, pp. 33-40, 2007.

[19] D. Sanchez, M. Tentori, and J. Favela, "Activity recognition for the smart hospital”, IEEE Intelligent Systems, vol. 23 (2), pp. 50-57, 2008.

[20] J. Favela, A.I. Martinez, M.D. Rodriguez, and V.M. Gonzalez, "Ambient computing research for healthcare: challenges, opportunities and experiences”, Computacion y Sistemas, vol. 12 (1), pp. 109-127, 2008. 
[21] J.E. Bardram, T. R. Hansen, and M. Soegaard, "AwareMedia - a shared interactive display supporting social, temporal, and spatial awareness in surgery", Computer Supported Cooperative Work, 2006.

[22] Bardram, J., T.R. Hansen, M. Mogensen, and M. Soegaard. "Experiences from real-world deployment of context-aware technologies in a hospital environment", in Ubicomp. 2006. Orange County, CA, USA.

[23] L.Wilcox, D. Morris, D. Tan, and J, Gatewood, "Designing patientcentric information displays for hospitals", CHI 2010, Atlanta, Georgia, USA.
[24] M. Skeels, and D.S. Tan, "Identifying opportunities for inpatient-centric technology", IHI 2010, Arlington, Virginia, USA.

[25] T. Bickmore, L. Pfeifer, and B. Jack, "Taking the time to care: empowering low health literacy hospital patients with virtual nurse agents", CHI 2009, Boston, MA, USA.

[26] L. Wilcox, S. Feiner, S. Restaino, S. Collins, and D. Vawdrey, "Designing inpatient technology to meet the medication information needs of cardiology patients", IHI 2012, Miami Florida, USA.

[27] http://www.youtube.com/watch?v=1edIODuCxes 